\title{
Essential phospholipids in fatty liver: a scientific update
}

This article was published in the following Dove Press journal:

Clinical and Experimental Gastroenterology

5 May 2016

Number of times this article has been viewed

\author{
Karl-Josef Gundermann' \\ Simon Gundermann² \\ Marek Drozdzik' \\ VG Mohan Prasad ${ }^{3}$ \\ 'Department of Pharmacology, \\ Pomeranian Medical University, \\ Szczecin, Poland; ${ }^{2}$ Department of \\ Radiology, Hospital Hohenlind, \\ Cologne, Germany; ${ }^{3}$ VGM Hospital \\ Institute of Gastroenterology, \\ Coimbatore, India
}

Aim: Although essential phospholipids (EPL) from soybean are often used in membraneassociated disorders and diseases, their high quality of purification and effects on prevalent liver diseases, especially on fatty liver diseases (FLDs) of different origin, are still widely unknown and a matter of continuous active research. The aim of this article is to review, discuss, and summarize the available results of EPL in the treatment of FLD.

Methods: Database research was carried out on Medline, Embase, Cochrane Library, countryspecific journals, and follow-up literature citations for relevant hepatogastroenterological articles published between 1988 and 2014. We searched for and reviewed only those papers that indicated minimum extraction amount of $72 \%$ (3-sn-phosphatidyl)choline from soybean as being necessary to treat patients with a considerable amount of 1,2-dilinoleoylphosphatidylcholine as a key component in EPL.

Results: EPL has a well-established mode of action, therapeutic effectiveness, and lack of toxicity, which ensures clinically relevant efficacy-to-safety ratio. It influences membrane-dependent cellular functions and shows anti-inflammatory, antioxidant, antifibrogenic, antiapoptotic, membrane-protective, and lipid-regulating effects. Due to its positive effects on membrane composition and functions, it accelerates the improvement or normalization of subjective symptoms; pathological, clinical, and biochemical findings; hepatic imaging; and liver histology. It is justified to administer EPL together with other therapeutic measurements in the liver.

Conclusion: Pharmacological and clinical results confirm the efficacy of EPL in the treatment of FLD.

Keywords: fatty liver disease, essential phospholipids, dilinoleoylphosphatidylcholine, membrane

\section{Introduction}

Nonalcoholic fatty liver disease (NAFLD) and alcoholic liver disease (ALD) are one and the same fatty liver disease (FLD) with an underlying multicausal origin, such as obesity, alcohol drinking, and diabetes mellitus, ${ }^{1}$ which are important public health problems, for which an established therapy is not yet available. This is a medical challenge as progressive course from simple steatosis to steatohepatitis and possible cirrhosis with development of liver cancer is known. ${ }^{1-3}$ Therapy of NAFLD is currently mainly directed at treating components of the metabolic syndrome. Diet and physical exercise are recommended as a basic universal approach. Some pharmacological agents show promising results, although on the basis of recent clinical trials no firm conclusions can yet be drawn. ${ }^{4}$ The situation is comparable with ALD. ${ }^{3}$ The nontoxic essential phospholipids (EPL) have been already widely accepted to be effective in
Correspondence: VG Mohan Prasad VGM Hospital Institute of Gastroenterology, Trichy Road, Rajalakshmi Mills Stop, Singanallur 64I005 Coimbatore, India

Tel +9l 9842204995

Emailvgmh2009@gmail.com
Clinical and Experimental Gastroenterology 2016:9 105-II7

105

Dovepress

http://dx.doi.org/10.2147/CEG.S96362 (c) 9 (i) 2016 Gundermann et al. This work is published and licensed by Dove Medical Press Limited. The full terms of this license are avalable at https://www.dovepress.com/
terms.php and incorporate the Creative Commons Attribution - Non Commercial (unported, v3.0) License (http:///creativecommons.org/licenses/by-nc/3.0). By accessing the work you herebby accept the Terms. Non-commercial uses of the work are permitted without any further permission from Dove Medical Press Limited, provided the work is properly attributed. For permission for commercial use of this work, please see paragraphs 4.2 and 5 of our Terms (https://www.dovepress.com/terms.php). 
various liver diseases. ${ }^{5,6}$ However, as a clinical overview of available results specifically in FLDs of different origin has not yet been published since 1988, it is the purpose of this article to evaluate the clinical efficacy and safety of EPL in these frequent liver diseases. The term EPL with its polyenylphosphatidylcholine (also called polyene phosphatidylcholine [PPC]) molecules indicates a well-defined, highly purified extract of the semen of soybeans (glycine max) with standardized contents of 72\%-96\% (3-sn-phosphatidyl) choline. The quantitatively and qualitatively dominating molecule in EPL is 1,2-dilinoleoylphosphatidylcholine (DLPC) (Figure 1), with up to $52 \%$ of the administered PCs. ${ }^{6}$ This high level of DLPC is the main difference between EPL/PPC and the typical phospholipids as well as PC consumed through diet and synthesized within the body. With a share of $\sim 1.3 \%$, highly unsaturated PCs with an additional, unsaturated fatty acid in the first position of the PC molecule are rare. ${ }^{7}$ By administering EPL, the amount of hepatic DLPC significantly increased. ${ }^{8}$

Phospholipids are known to form the double layer of cellular and subcellular membranes and precondition their fluidity and biological activity. The efficacy of EPL in the therapy of liver diseases is confirmed not only by the ability of DLPC to be incorporated into damaged sections of membranes, which improves hepatic regeneration and replaces endogenous, less unsaturated PC molecules but also by its ability to increase membrane fluidity and functioning. With regard to in vitro and animal investigations, EPL influences membrane-dependent cellular functions and shows antioxidant, anti-inflammatory, antifibrotic, apoptosis-modulating, regenerative, membrane-repairing and -protective, cellsignaling and receptor-influencing, and lipid-regulating effects. ${ }^{6}$

\section{Materials and methods}

Database research was carried out on Medline, Embase, Cochrane Library, country-specific journals, and follow-up literature citations for relevant hepatogastroenterological articles published between 1988 and 2014. In a two-step process, we first used combinations of keywords including NAFLD, ALD, essentiale, ESLs, polyenylphosphatidylcholine, polyene phosphatidylcholine, dilinoleoylphosphatidylcholine, phosphatidylcholine, and therapy. In the second step, we reviewed those papers that indicated minimum extraction amount of $72 \%$ PC from soybean as being necessary to treat patients with a considerable amount of DLPC as a key component in EPL (for details see later), or that were based on the trade name Essentiale ${ }^{\circledR}$. Studies with less purified PC from soybean or with PC from other provenience were excluded. All papers that were reported in different languages other than English were completely translated into English. Of the 83 clinical studies in total, 25 representative ones regarding the causes of FLD, study design, and investigated variables were selected, including one case report study and seven double-blind studies (Table 1).

\section{Results}

\section{Clinical efficacy of EPL in NAFLD}

Fifty-three clinical studies about EPL and NAFLD have been published since 1988, of which 45 were published since 2000. Twenty-eight, mostly randomized studies, were open controlled and three double-blind. Dosage of EPL and treatment duration varied as a rule between $1.05 \mathrm{~g} / \mathrm{d}$ and $1.8 \mathrm{~g} / \mathrm{d}$ per os and lasted from 4 weeks to $>24$ months, in most studies 3-6 months with $1.8 \mathrm{~g}$ of EPL/d. Therapy started in some studies with 500-1,000 mg EPL iv over 10 days to 4 weeks, before continuing with oral EPL

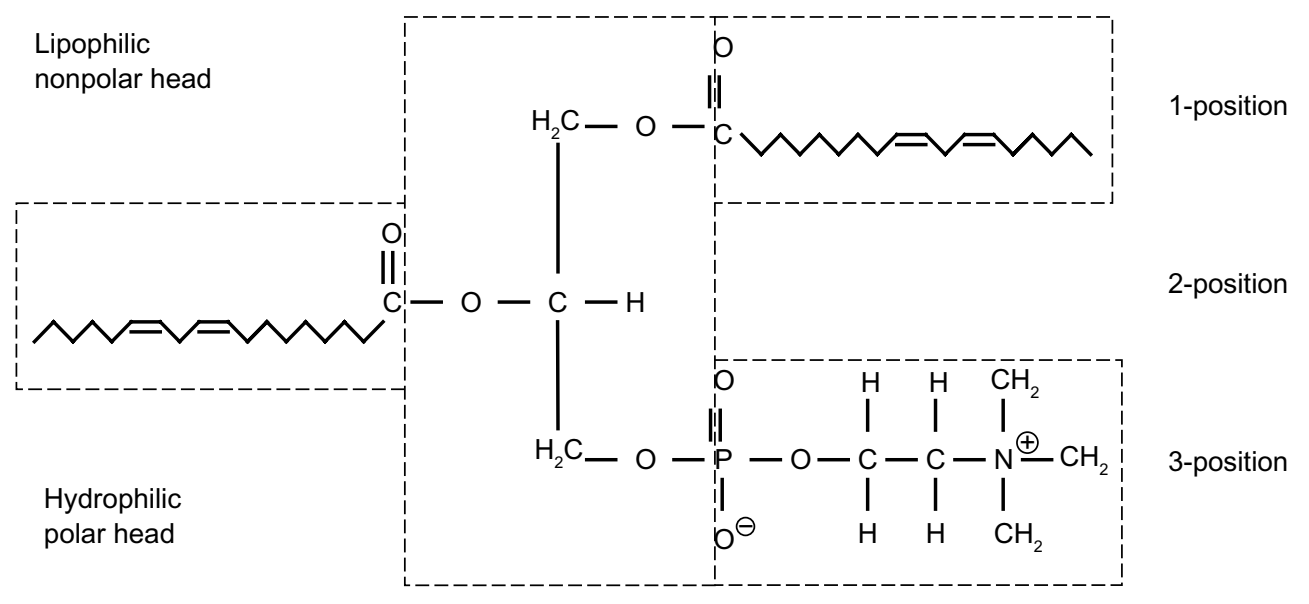

Figure I I,2-Dilinoleoylphosphatidylcholine (DLPC), the main active ingredient in EPL. Abbreviation: EPL, essential phospholipids. 


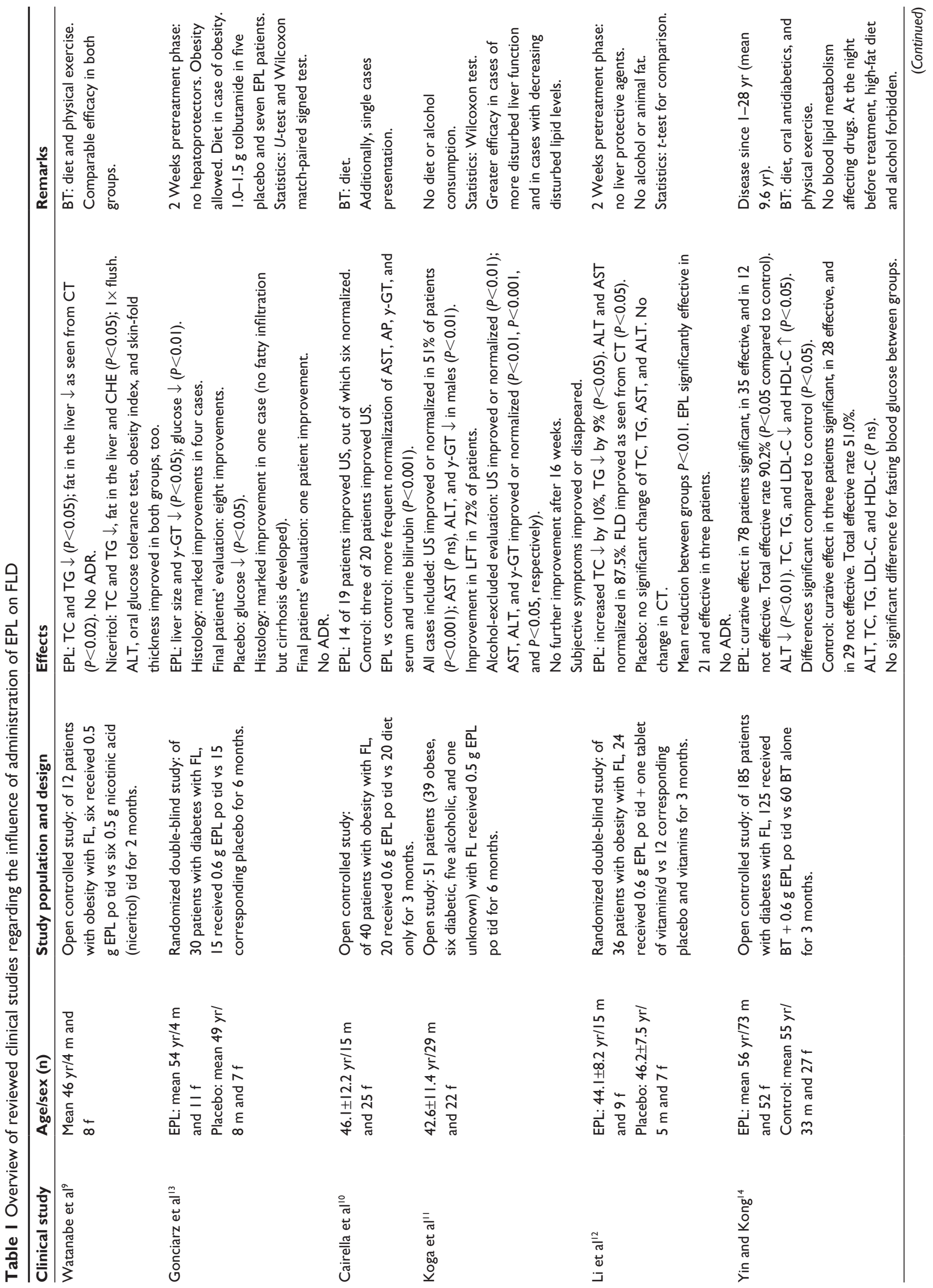



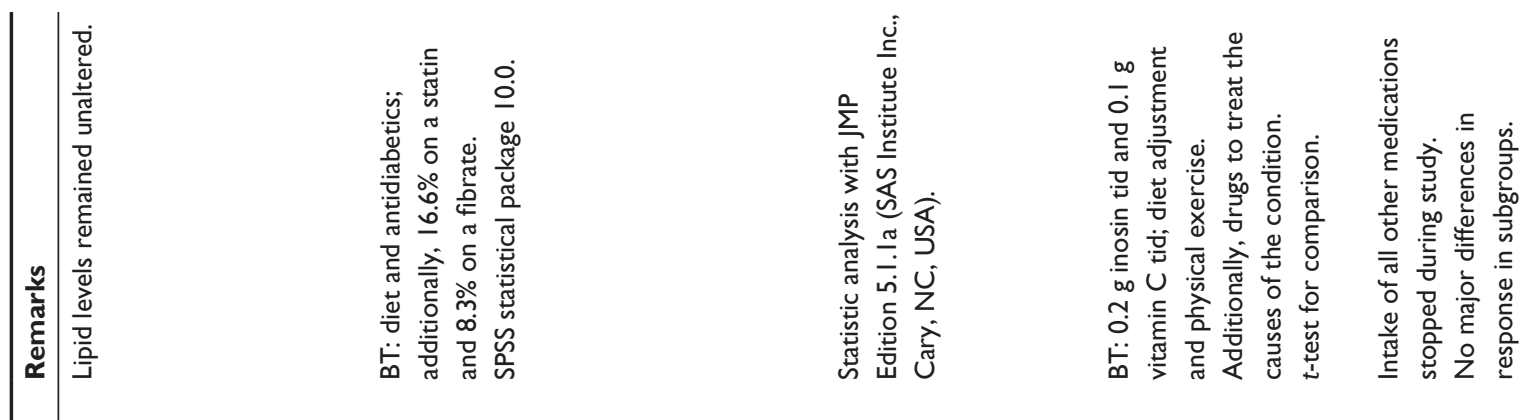

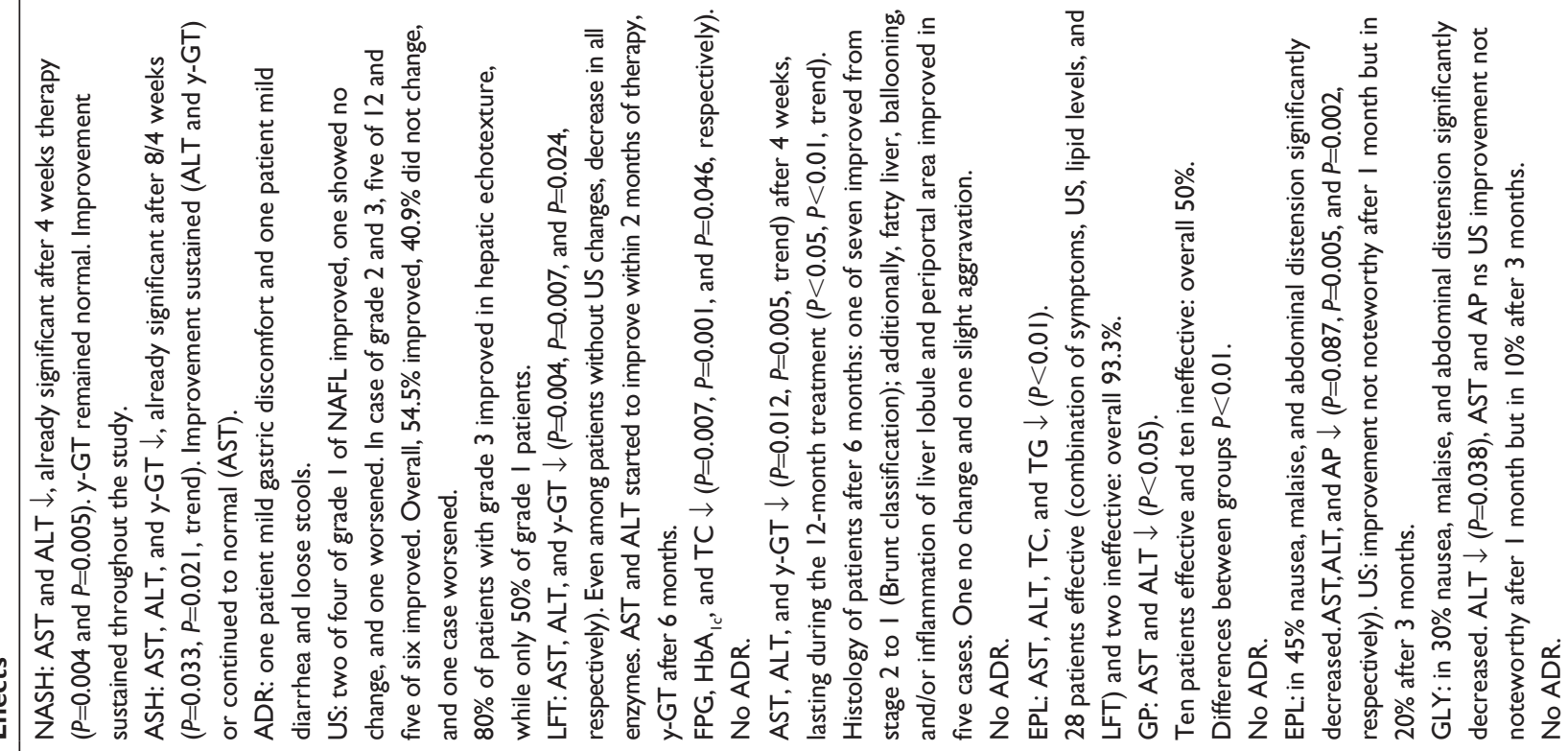
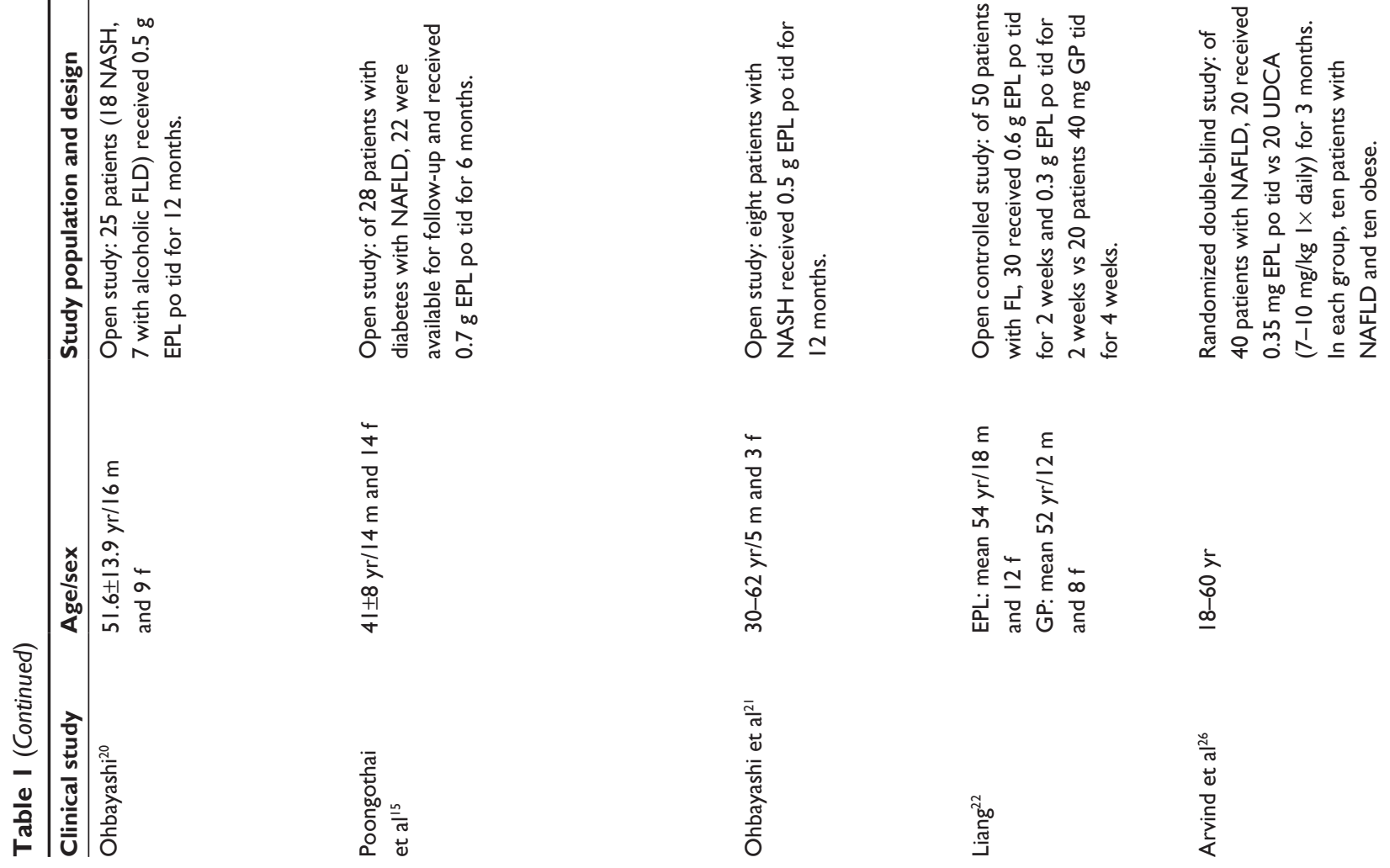

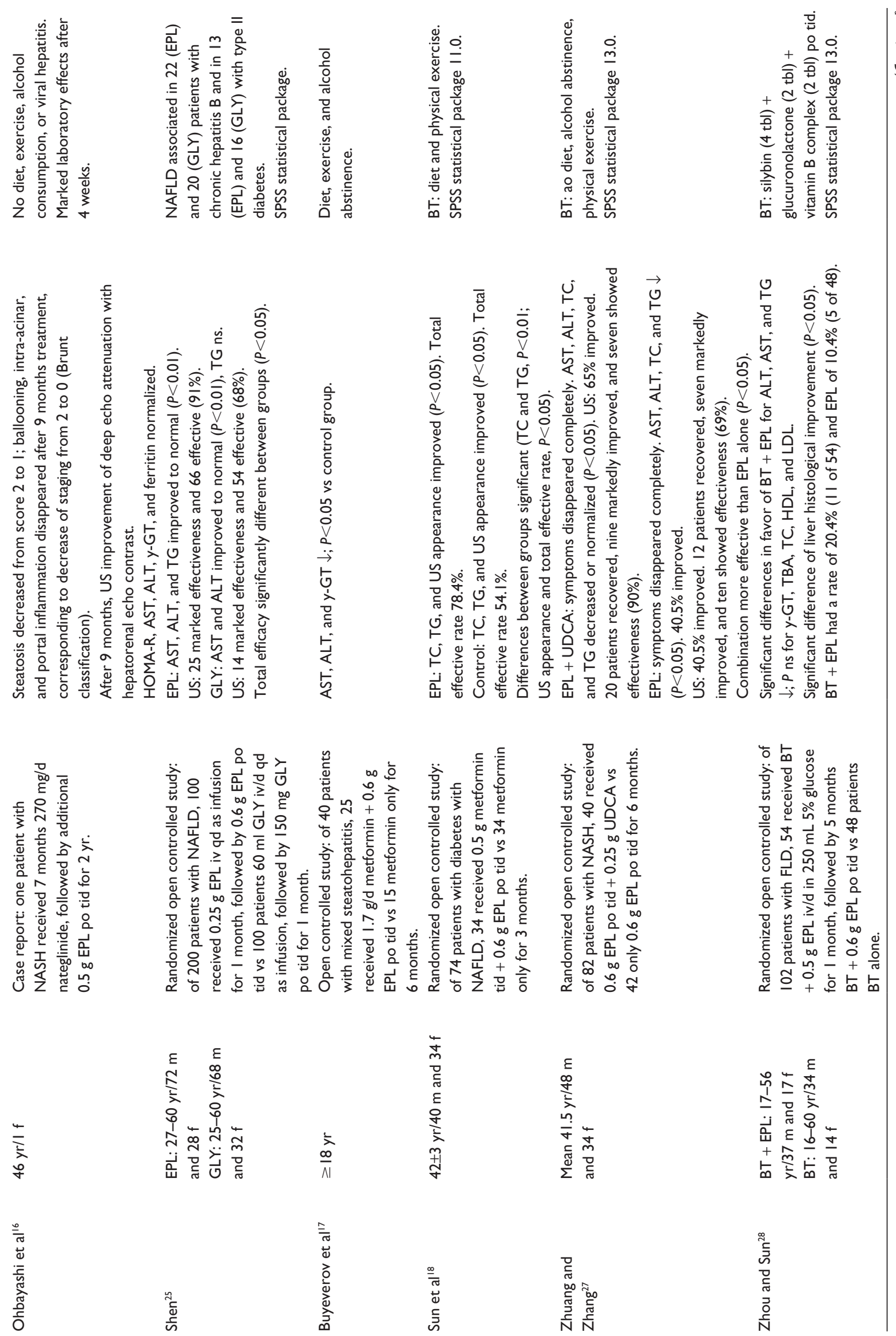

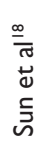

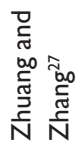

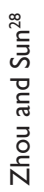



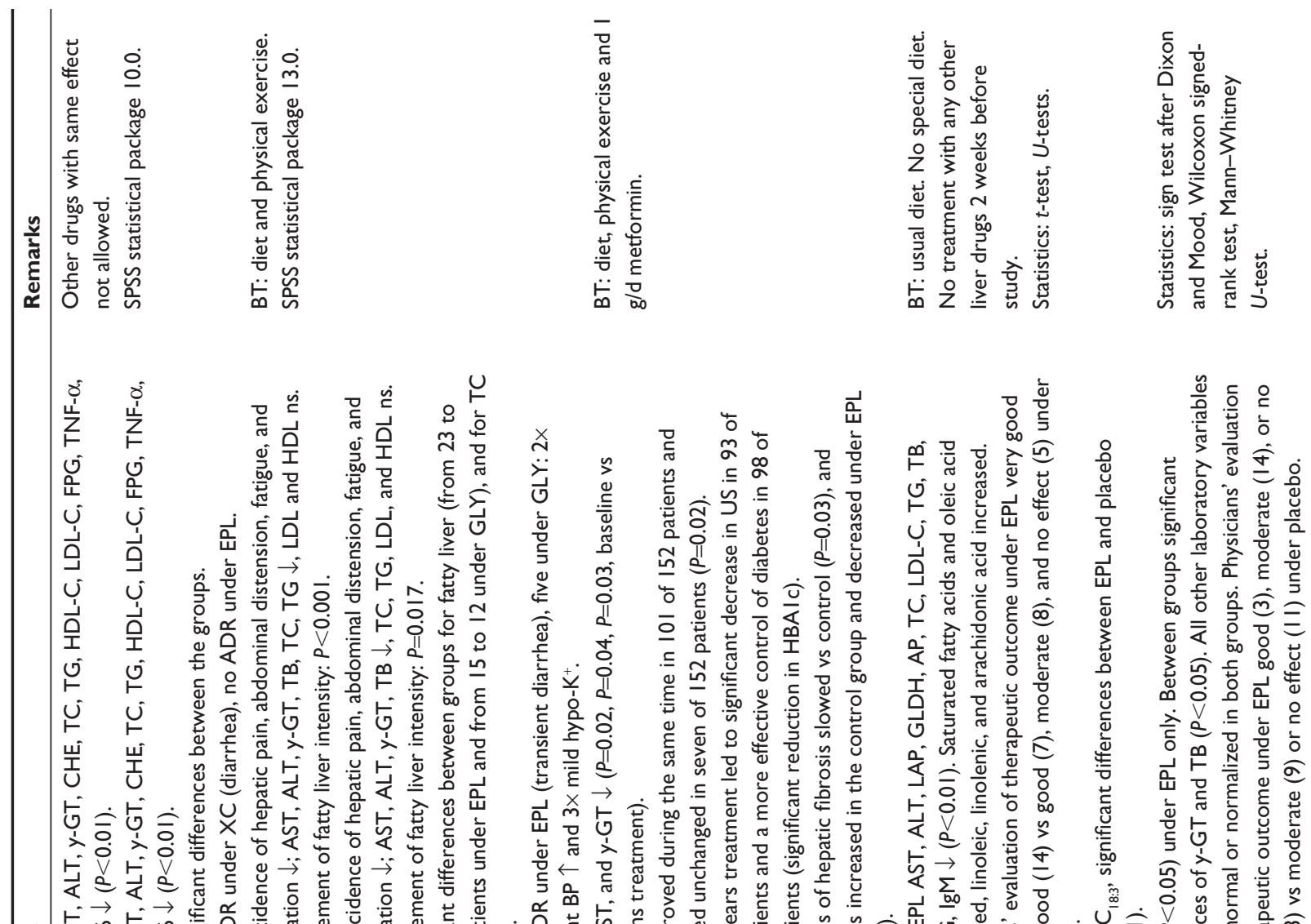

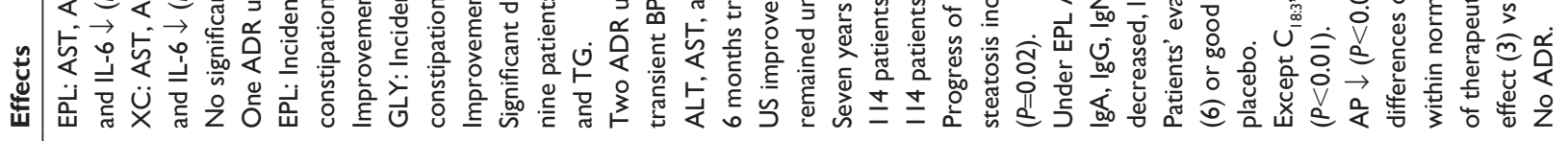
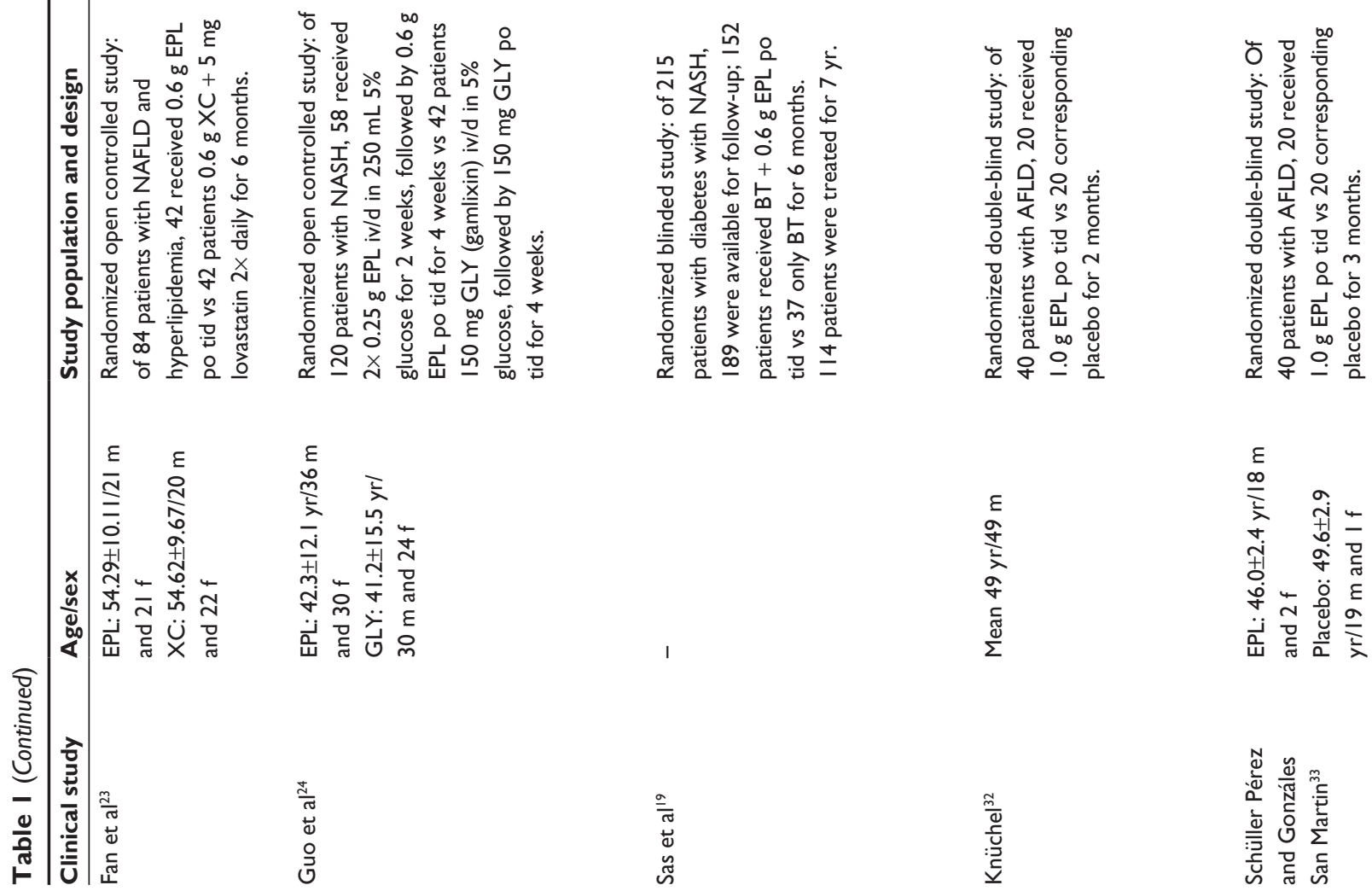


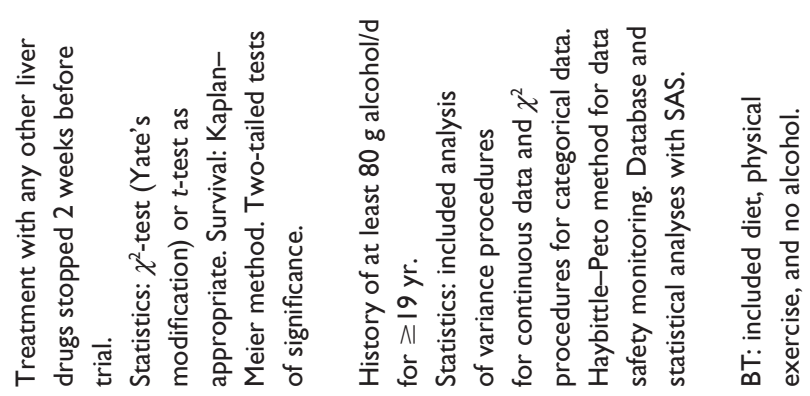

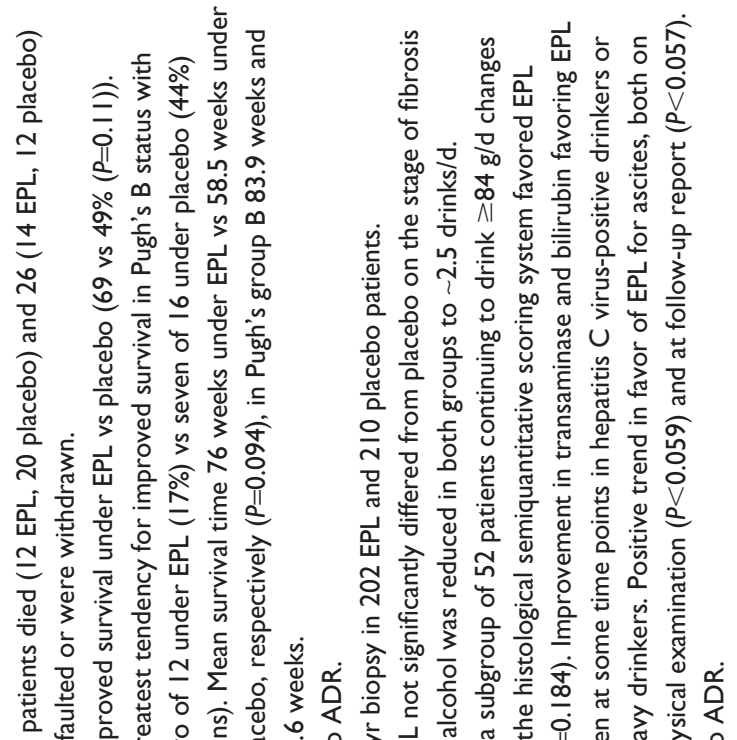

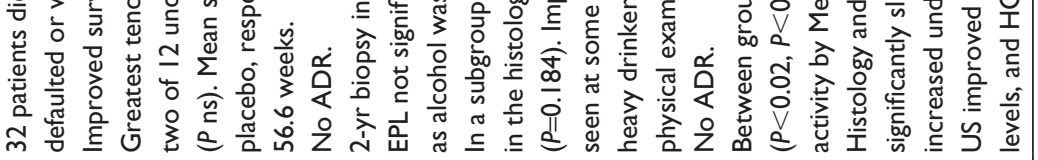
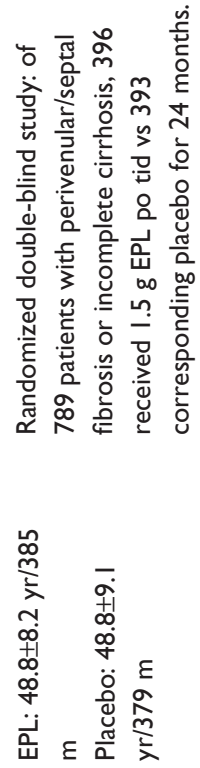

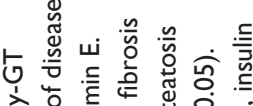

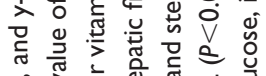

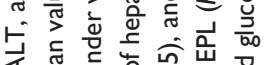

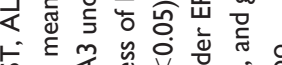

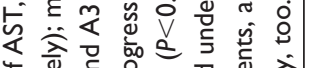

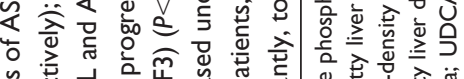

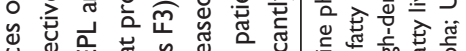

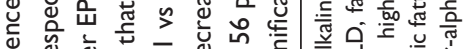

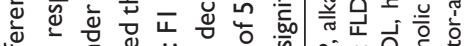

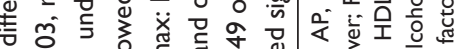

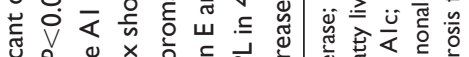

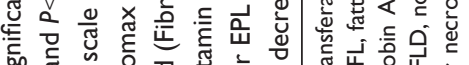

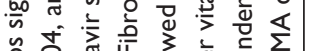

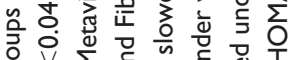

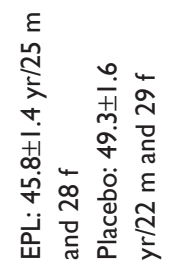

$\frac{0}{c}$
$\stackrel{0}{0}$
0
$\frac{0}{0}$
$\frac{0}{0}$

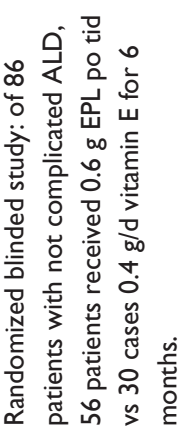

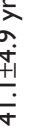

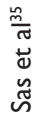

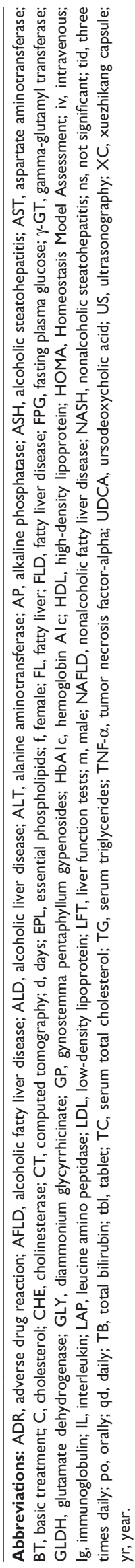


administration; one study was done with $500 \mathrm{mg}$ EPL iv for 30 days only. NAFLD and its more severe form, nonalcoholic steatohepatitis (NASH), were mainly induced by obesity and type II diabetes mellitus but partly also associated with hyperlipidemia, arterial hypertension, coronary heart disease/atherosclerotic cardiosclerosis, chronic viral hepatitis, cholecystitis/cholelithiasis, hypothyroidism, and pregnancy, or with mixed forms of these diseases/disorders. According to the available studies, analysis of subjective symptoms, clinical findings, biochemical and imaging data, and histology was possible.

In the first clinical study from Japan, six nonalcoholic and nondiabetic patients with fatty liver due to obesity were successfully treated with a low-calorie diet, physical exercise, and $1.5 \mathrm{~g}$ EPL/d for 8 weeks and compared to six patients under the same diet, physical exercise, and a nicotinic acid derivative prodrug. ${ }^{9}$ It was found that computed tomography (CT) determined liver fat accumulation significantly improved with EPL $(27 \pm 18 \mathrm{HU}$ to $54 \pm 8 \mathrm{HU}$ indicating the decrease in intensity of steatosis).

Cairella et $\mathrm{al}^{10}$ from Italy evaluated the efficacy of EPL in therapy of hepatobiliary dysfunction when administering $1.8 \mathrm{~g}$ of EPL/d for 3 months. The study group and control comprised 20 cases each with a body mass index of $35.34 \pm 4.89 \mathrm{~kg} / \mathrm{m}^{2}$. The excretory parameters, total bilirubin, alkaline phosphatase, and y-GT, were normalized in all patients of the EPL group but remained increased in $15 \%-35 \%$ of the control patients. In the EPL group, the ultrasonographic picture clearly improved in 14 and normalized in six cases, while it only slightly changed in three cases of the control group. Koga et al ${ }^{11}$ confirmed the continuous, EPL-induced improvement or normalization of the ultrasonic picture in 39 patients with fatty liver due to obesity. Bright liver was reduced, and decrement of backward echocardiogram and the obscurity of the intrahepatic venogram were decreased.

In 2000, a randomized double-blind study by Li et al ${ }^{12}$ reported about the positive influence of $1.8 \mathrm{~g}$ of EPL/d on total cholesterol (TC), triglycerides (TG), and transaminases, supported by significant improvement of the fatty liver (CT scan).

Experience with EPL in patients suffering from maturityonset diabetes mellitus and fatty liver started with a randomized, placebo-controlled double-blind study. ${ }^{13}$ Thirty patients were instructed to reduce diet to $1,200 \mathrm{kcal} / \mathrm{d}$ containing $1 \mathrm{~g} / \mathrm{kg}$ protein. There were five patients in the placebo and seven patients in the EPL group who were treated with $1.0-1.5 \mathrm{~g}$ tolbutamide per day. Half of the patients were additionally given $1.8 \mathrm{~g}$ of EPL/d, the second half a corresponding placebo. During the 6-month treatment phase, liver size hardly changed in the patients of the placebo group, but there was a significant reduction in liver size in the EPL group. The histological examination of the liver biopsy yielded corresponding results: marked improvement was observed in four patients treated with EPL and only in one patient of the placebo group. Although no fatty infiltration was seen in the posttreatment liver biopsy of this patient, he developed the feature of progression to cirrhosis. Also in one patient treated with EPL, whose fatty infiltration was more pronounced at the second biopsy, morphological features of developing discrete fibrosis in portal tract were observed. Altogether, there were seven patients in the EPL group and four under placebo including the patient with developing cirrhosis, whose steatosis improved histologically during the study. Additionally, y-GT was significantly lowered after 1 month, 3 months, and 6 months of EPL therapy, whereas in the control group, the deviations were not statistically significant. Transaminases and bilirubin were within normal range throughout the study.

Since this double-blind trial, seven further clinical studies confirmed the influence of EPL on diabetes mellitusassociated fatty liver. ${ }^{14-20}$ Yin and Kong ${ }^{14}$ treated 185 patients with standard diet, oral antidiabetics (not specified) plus physical exercise, and 125 of these patients were treated additionally with $1.8 \mathrm{~g}$ of EPL/d for 3 months. Alanine aminotransferase (ALT), serum TC, and TG, low-density lipoprotein- and high-density lipoprotein-cholesterol had significantly improved or normalized within the EPL group, the lipid variables also between the groups. The improvements were seen in $90.2 \%$ of the EPL cases and in $51 \%$ of the controls. This difference was statistically significant $(P<0.05)$. Fasting blood sugar values improved in both groups, but the difference between the groups was not significant. Twentytwo patients from the study of Poongothai et $\mathrm{al}^{15}$ received, besides a standard diet, antidiabetics depending on the severity of the glycemic level: $22.7 \%$ were on metformin therapy, $22.7 \%$ on sulfonylurea, $45.5 \%$ on combination of both, and $9 \%$ on insulin therapy. EPL $2.1 \mathrm{~g} / \mathrm{d}$ per os was additionally given for 6 months. Ultrasonographic improvement was seen in $54.5 \%$ of patients, $40.9 \%$ did not show any change, and one case worsened. Hepatic echotexture improved in $80 \%$ of subjects with severe diffusion fine echos in hepatic parenchyma, and in $50 \%$ of those with slight changes. Transaminases and y-GT improved too. Ohbayashi et al $^{16}$ treated a female patient with NASH with nateglinide as insulin secretagogue for 7 months, followed by an additional $1.5 \mathrm{~g}$ of EPL/d for a total of 2 years. The Homeostasis Model Assessment, (an 
index of insulin resistance) normalized, same as hepatorenal echo contrast, transaminases, $y-G T$, and ferritin. The beneficial effect continued over the 24 months of study. Liver biopsy, evaluated using Brunt's criteria, improved from stage two to zero after 9 months of EPL administration; steatosis decreased, and ballooning, intra-acinus inflammation, and portal tract inflammation disappeared.

During the last 8 years, three studies investigated the combination of metformin and EPL on patients with diabetes, and the authors observed the same positive effects on aspartate aminotransferase, y-GT, blood lipids, and ultrasonic appearance. ${ }^{17-19}$ According to the latest study, hepatic fibrogenesis slowed down in the EPL group versus control $(P=0.03)$, and steatosis decreased under EPL and increased in the control group (difference between groups $P=0.02$ ). ${ }^{19}$

Besides the abovementioned study, Ohbayashi ${ }^{20}$ and Ohbayashi et $\mathrm{al}^{21}$ confirmed with further 18 and eight patients with NASH that long-term administration of EPL continues to improve hepatic function until completion of the study. First, significantly decreased transaminase levels were seen within 4-week treatment and continued over a 12-month therapy. ${ }^{20}$ Of the seven patients of the second study with a biopsy before and after 6-month treatment, one had a significantly improved histological picture, four others had some improvements of steatosis, ballooning, inflammation of the liver lobe, and/or of the periportal area, one had no histological change, and one had a slight aggravation of the picture. ${ }^{21}$

During the last years, EPL was compared to other compounds, discussed to be effective in NAFLD. The randomized, open controlled studies compared EPL to Gynostemma pentaphyllum gypenosides, to an extract from red yeast rice (Xuezhikang; each capsule contains $2.5 \mathrm{mg}$ of lovastatin), or to diammonium glycyrrhizinate..$^{22-25}$

Compared to the gypenosides of G. pentaphyllum, EPL showed better relief of the clinical symptoms, improvement in serum TC and TG, ultrasound picture, and liver function. EPL showed obvious effects in $93.3 \%$ of the cases. ${ }^{22}$ The randomized, open controlled studies from Fan et $\mathrm{al}^{23}$ and Guo et $\mathrm{al}^{24}$ showed no differences between the extracts of the control group and EPL in the significant recovery of liver function. However, EPL was more effective on serum lipid levels and fatty liver severity than diammonium glycyrrhizinate. Especially, the proportion of serious fatty liver changed significantly in the EPL group from 23 to nine patients versus 15 to 12 patients in the control group. ${ }^{24}$ Comparison of liver ultrasonography for the number of markedly effective and effective improvements showed significant differences in favor of EPL versus diammonium glycyrrhizinate. ${ }^{25}$
The abovementioned randomized, open controlled studies $^{22-25}$ are added by a double-blind one, which compared 3-month therapy of $1.05 \mathrm{~g}$ of EPL/d with $7-10 \mathrm{mg} / \mathrm{kg}$ ursodeoxycholic acid (UDCA) per day. ${ }^{26}$ Ten patients of each group were diabetics with NAFLD, and the remaining were obese individuals (body mass index $>30 \mathrm{~kg} / \mathrm{m}^{2}$ ) with NAFLD. Patients with cardiovascular disease, liver disease other than hepatosteatosis, complications of diabetes including ketoacidosis, pregnant and lactating women, and patients with a history of alcohol consumption were excluded from the study. Intake of all other medications was stopped during the evaluation period. EPL was more effective than UDCA. Forty-five percent of patients on EPL expressed satisfaction with therapy in terms of a significant reduction in symptoms (nausea, malaise, and abdominal distension) at the end of therapy as opposed to $30 \%$ of patients on UDCA. No noteworthy ultrasonic changes were seen after 4 weeks, but after 12 weeks, $20 \%$ of patients on EPL and $10 \%$ of patients on UDCA displayed some degree of ultrasonic improvement. ALT and AP were significantly improved in the EPL arm. In contrast, only ALT fell significantly in subjects on UDCA but not aspartate aminotransferase or AP. There were no major differences in the response of the diabetic and obese subgroups.

The clinical picture of EPL and NAFLD is completed by studies from the beginning of this decade, which investigated a combination therapy of EPL with other drugs versus EPL therapy alone..$^{27,28}$ In these two randomized, open controlled studies, the combination of EPL with UDCA in patients with NASH and the combination of EPL with silybin, glucuronolactone, and vitamin B complex in patients with fatty liver were more effective than EPL alone.

\section{Clinical efficacy of EPL in ALD}

Thirty clinical studies about EPL and ALD have been published since 1988, and 17 since 2000. Twelve, partly randomized studies were open controlled, one single-blind, and two double-blind. Indications were either not further specified or included different types of ALD (number of studies [n] $=10)$, alcoholic fatty liver $(n=11)$, acute $(n=1)$ or chronic $(n=6)$ alcoholic hepatitis, chronic active alcoholic hepatitis or liver cirrhosis $(n=1)$, or patients with alcoholic perivenular fibrosis to incomplete cirrhosis $(n=1)$ were investigated. In most studies, patients were advised to stop alcohol intake during the observation period. With the exception of two clinical trials (for more details see later), dosage of EPL was $1.8 \mathrm{~g} / \mathrm{d}$ per os, and treatment duration varied from 4 weeks to 6 months. In some of the studies (with higher activity of 
the inflammatory process in the liver), therapy started with 500-1,000 mg EPL/d iv, followed by 1,800 mg EPL/d per os. Three studies were done with 500-1,000 mg EPL/d iv over 4 weeks without peroral EPL administration.

$\mathrm{Hu}$ et $\mathrm{al}^{29}$ published a systematic review of six randomized double-blind studies in the treatment of ALD and FLD. ${ }^{29}$ According to two independently evaluating persons, the methodological quality of these studies was high enough to be included for evaluation. All studies compared the effects to placebo. Two studies were in the frame of our database research, ${ }^{30,31}$ two were older, ${ }^{32,33}$ and in two studies patients suffered from NAFLD. ${ }^{12,13}$

Only one study reported on mortality rate, which was $22.6 \%$ in the treatment group and $39.2 \%$ in the control group. ${ }^{30}$ The criteria of efficacy of four trials of the systematic review with 146 patients were remission of clinical symptoms, signs, and biochemical variables (overall clinical efficacy). ${ }^{12,13,32,33}$ The response rate was 21 of 24 in the treatment group and three of 12 in the control group. A meta-analysis of the four studies showed an efficacy rate of $83.5 \%$ in the treatment group and $41.7 \%$ in the control group $(P=0.03) .{ }^{29}$ This suggests that in every 100 patients with ALD/FLD treated with EPL, 41 will benefit and respond to the treatment. Additionally, a total of 923 patients in three trials could be included for analysis of histology. ${ }^{13,30,31}$ According to the meta-analysis, EPL did not improve the patients' histology $(P=0.07)$, but histological worsening was prevented $(P=0.02)$.

Serious adverse events were not reported in the six studies. The randomized, prospective double-blind trials from Panos et $\mathrm{al}^{30}$ and Lieber et $\mathrm{al}^{31}$ were both performed according to Good Clinical Practice and differed from the older double-blind studies ${ }^{32,33}$ by the attempt to increase the efficacy of EPL by increasing dosage and duration of treatment. In the study by Panos et al, ${ }^{30} 53$ patients received $6 \mathrm{~g}$ EPL daily and 51 patients a corresponding placebo over a period of 2 years. A total of 46 patients completed the trial (EPL: $n=27$; placebo: $n=19$ ). Among the dropouts, 12 patients of the EPL group and 20 of the control group (31\% and 51\% of those patients who entered the trial and did not default) died. The survival curves, constructed by the Kaplan-Meier method, showed a notable trend toward improved survival by EPL as compared to placebo, although not being significant $(P=0.086)$. Patients with Child Pugh B score usually carry a higher death risk than those in classification A. Taking into account classification $\mathrm{B}$ only, the difference was more pronounced: two of the 12 EPL-treated patients died $(17 \%$ of those who entered, $25 \%$ of those who did not default) in comparison with seven of the 16 control patients $(44 \%$ of those who entered, $64 \%$ of those who did not default) (not significant). The mean survival in this subgroup was 83.9 weeks (EPL) and 56.6 weeks (placebo), respectively.

In the study by Lieber et al, ${ }^{31} 789$ heavy drinkers of 20 Veterans Affairs Medical Centers/USA with a history of daily average alcohol consumption of 16 drinks per day (one drink $=14 \mathrm{~g}$ of alcohol) for 19 years and with the evidence of perivenular or septal fibrosis or incomplete cirrhosis were randomly assigned to receive daily either $4.5 \mathrm{~g}$ EPL or placebo for up to 4-6 years. Two-year biopsy was completed in 412 patients. EPL did not significantly differ from placebo in its effect on the stage of fibrosis as the main outcome as alcohol intake was unexpectedly reduced in both groups to $\sim 2.5$ drinks per day as a result of a new "brief intervention" approach. Accordingly, there was no more progression of the fibrosis and therefore no way to test whether EPL could oppose such a progression except in a subgroup of patients who were still consuming six or more drinks a day and showed beneficial effects against fibrosis. Ascites, an important secondary clinical measure of liver disease, was also less frequently observed during follow-up of EPL-treated patients. Furthermore, improvements in aminotransferases and bilirubin favoring EPL were seen at some time points in the subgroups of drinkers who were positive for hepatitis $\mathrm{C}$ virus and in nondrinkers. ${ }^{34}$

In a randomized single-blind clinical study from Sas et al, ${ }^{35}$ 86 patients with uncomplicated ALD were adequately controlled by diet and abstinent from alcohol and followed a basic treatment scheme, including diet, physical regimen, and abstinence from alcohol. Fifty-six of these patients were additionally treated with $1.8 \mathrm{~g}$ of EPL/d for 6 months, and 30 patients of the control group with $400 \mathrm{mg}$ vitamin E per day.

Basic treatment plus vitamin $\mathrm{E}$ led to less reduction of transaminase levels than basic treatment plus EPL. Moreover, the mean value of disease activity evaluated by Metavir scale was after treatment A1 (mild activity) in the EPL group and A3 (severe activity) in the control group. Ultrasound studies revealed significant improvement in the hepatic echostructure in 49 of the 56 patients of the investigational group. Liver biopsy and Fibromax test showed that the additional EPL administration significantly slowed the progress of hepatic fibrosis to F1 (portal fibrosis without septa) versus F3 (numerous septa without cirrhosis) in the control group. Steatosis significantly increased in the vitamin E group and decreased in the EPL group. Additionally, the authors reported about significant reduction of glucose, insulin levels, and insulin resistance index. 
According to the results of a meta-analysis of nine randomized, placebo-controlled double-blind studies on the clinical efficacy of EPL in 409 hospitalized patients, who suffered from chronic liver disease (chronic active hepatitis or fatty degeneration of the liver), the overall effect was in favor of EPL $(P<0.0001)$, with the mean difference of the responder rates being $26.6 \%$ better for EPL. ${ }^{36}$

\section{Discussion}

NAFLD is the best investigated indication of EPL in liver diseases during the last 12 years. Not much therapeutic options for NAFLD are accepted until today besides correction of obesity with hypocaloric diets and physical exercise and controlling hyperglycemia with diet, insulin, or oral hypoglycemic agents. Therefore, any therapeutic intervention that targets fat accumulation in the liver and ameliorates hepatic histology would be of great benefit. ${ }^{37}$ Not much has changed therapeutically since this statement was published in 2006, but EPL is one of the drugs under discussion with significant positive effects on NAFLD (Table 1). Because of its membranous, antioxidative, and antifibrotic effects, administration of EPL in NAFL and NASH is pathogenetically justified. An additional important reason for the efficacy of EPL in NAFLD was recently published by Ling et al, ${ }^{38}$ who showed that decreased hepatic PC-to-phosphatidylethanolamine ratio in cellular membrane is a predictor of NAFLD and survival following partial hepatectomy. EPL is able to re-improve this disturbed ratio. As mentioned, besides low-calorie diets and physical loads, therapy of NAFLD should be directed at treating components of metabolic syndrome, which may be additionally beneficial for the liver. The International Diabetes Federation consensus' worldwide definition of the metabolic syndrome from 2006 is central obesity (defined as waist circumference with ethnicity-specific values) and any two of the following criteria: raised TG, reduced highdensity lipoprotein cholesterol, raised blood pressure, or raised fasting plasma glucose (or previously diagnosed type 2 diabetes). ${ }^{39}$ This definition includes TG and HDLcholesterol, both criteria, which have been successfully treated by EPL, too..$^{40,41}$

ALD is also intensively investigated with EPL. ${ }^{5}$ The investigations from Okiyama et $\mathrm{al}^{42}$ exemplarily show that the mode of action of EPL in ALD is already quite well understood. However, clinically EPL is not as well investigated as pharmacologically. Though the authors found four double-blind and one single-blinded studies for analysis, two are older ones and two were done with significantly higher doses than recommended in the marketed form. ${ }^{30,31}$ The patients with acute alcoholic hepatitis had a daily intake of 12 capsules with a total of $6 \mathrm{~g}$ of EPL over 2 years. ${ }^{30} \mathrm{It}$ is questionable whether alcohol-abusing patients with basically well-known low compliance take 12 capsules every day for 2 years. Apart from that, it has been shown that the compliance of patients with ALD can be increased by a good intervention approach, which leads to alcohol abstinence or at least clear reduction of the alcohol consumption, with corresponding improvements of the hepatic status. ${ }^{43}$ Therefore, the first request is always absolute alcohol abstinence and then to consider drug treatment such as EPL, the latter especially to include from the beginning of treatment those cases who have problems to stop drinking. As there was a trend for higher survival of the patients with acute alcoholic hepatitis under EPL, especially of those with Child Pugh B disease severity, ${ }^{30} \mathrm{a}$ further promising double-blind study is recommended with such patients but this time with a lower daily dosage of EPL and a shorter duration of treatment, for example, with $1.8 \mathrm{~g}$ of EPL/d over 1-year treatment.

The Veterans Affairs Cooperative Study had additional shortcomings besides too high daily EPL dose and the long duration of treatment to show EPL efficacy. ${ }^{31}$ Planning the study, it was estimated that in the group of long-term heavy drinkers with early stages of fibrosis, the natural progression rate would be $30 \%$ over 24 months under conditions of continued drinking. However, the observed natural progression rate based on the placebo group outcome was $20 \%$, with a comparable rate of regression. The slower progression rate was supposed to be related to the reduction in drinking that occurred. Therefore, most patients did not meet the protocol entry definition of heavy drinking (six or more drinks per day) during the follow-up. Even in the subgroup of heavy drinkers, the predicted progression rate of $30 \%$ did not occur. A second drawback was that more than double the rate of patients anticipated in the sample size adjustment withdrew or were lost to follow-up before the 24-month biopsy.

On the other hand, most of the analyzed clinical studies on EPL in NAFLD and ALD have their shortcomings: too low number of patients, incomplete methodological description including unclear statistical evaluation, varying dosages of EPL, data published in secondary papers, or others. Therefore, further randomized double-blind clinical studies with higher number of patients and of comparable histology or imaging status are necessary to regard the administration of EPL as a standard treatment. Until today, only diet and physical exercise in NAFLD and alcohol abuse in ALD are worldwide accepted standard procedures, though even the 
exact optimum diet and kind and duration of physical exercise are not yet known.

To sum it up, most studies from different countries show some advantage of EPL versus other investigated drugs or if added to other drugs. This is indicative of the usefulness of EPL in the discussed liver diseases. This estimation is substantiated by the knowledge that all liver diseases are associated with cellular membrane damages and by the high number of positive pharmacological studies. ${ }^{6}$

\section{Conclusion}

EPL accelerates the improvement or normalization of subjective symptoms and pathological findings, such as pain in the right hypochondrium, dyspeptic symptoms, and hepatomegaly, in NAFLD and ALD. The findings are supported by imaging procedures, such as ultrasonography, CT, and Fibromax; by biochemical markers of hepatic cytolysis, detoxification, excretion, synthesis, and clearance; and by liver histology. No relevant side effects were reported. Additional adequately conducted randomized clinical trials on EPL versus placebo or other active treatment are recommended to investigate the synergistic effects of EPL with other liver drugs.

\section{Acknowledgment}

The authors wish to acknowledge the contribution of Dr Anant Patil for assistance with manuscript preparation.

\section{Disclosure}

Financial support for technical assistance in manuscript writing and for publication support was provided by Abbott Healthcare Pvt Ltd, India. The authors report no other conflicts of interest in this work.

\section{References}

1. Völzke H. Multicausality in fatty liver disease: is there a rationale to distinguish between alcoholic and non-alcoholic origin? World $J$ Gastroenterol. 2012;18(27):3492-3501.

2. Michelotti GA, Machado MV, Diehl AM. NAFLD, NASH and liver cancer. Nat Rev Gastroenterol Hepatol. 2013;10(11):656-665.

3. Orman ES, Odena G, Bataller R. Alcoholic liver disease: pathogenesis, management, and novel targets for liver therapy. J Gastroenterol Hepatol. 2013;28(Suppl 1):77-84.

4. Verbeek J, Cassiman D, Lannoo M, et al. Treatment of non-alcoholic fatty liver disease: can we already face the epidemic? Acta Gastroenterol Belg. 2013;76(2):200-209.

5. Kuntz E, Kuntz HD, editors. Hepatology. 3rd ed. Heidelberg: Springer; 2008:894-896.

6. Gundermann KJ, Kuenker A, Kuntz E, Drozdzik M. Activity of essential phospholipids (EPL) from soybean in liver diseases. Pharmacol Rep. 2011;63(3):643-659.

7. Oette K, Kühn G, Römer A, Niemann R, Gundermann KJ, Schumacher R. The absorption of dilinoleoyl-phosphatidylcholine after oral administration [Resorption von Dilinoleoyl-Phosphatidylcholin nach oraler Gabe]. Drug Res. 1995;45(11):875-879. German.
8. Lieber CS, Robins SJ, Li J, et al. Phosphatidylcholine protects against fibrosis and cirrhosis in the baboon. Gastroenterolgy. 1994;106(1):152-159.

9. Watanabe A, Kobayashi M, Morishita N, Nagashima H. Multimodal treatment resulting in rapid improvement of fatty liver in obese patients. Curr Ther Res. 1988;43(2):239-246.

10. Cairella M, Callisto F, Godi R, Marchini G. Polyunsaturated phosphatidylcholine combined with vitamin B complex in the treatment of patients with disorders of the hepatobiliary function caused by unbalanced nutrition [La fosfatidilcolina polinsatura associata a complesso vitaminico B nel trattamento del danni della funzione epato biliare da alimentazione squilibrata]. Clin Ter. 1989;131(4):237-246. Italian.

11. Koga S, Irisa T, Miyata Y, et al. Clinical progress of 51 fatty liver cases analyzed by liver function tests and ultrasonic screening and results of EPL administered cases. Prog Med. 1991;11(7):1891-1899. Japanese.

12. Li JH, Chen XY, Zhong CF, Min J. A randomized controlled study of essential phospholipids (Essentiale capsules) in the treatment of fatty liver. Infect Dis Info. 2000;13(4):180-181. Chinese.

13. Gonciarz Z, Besser P, Lelek E, Gundermann KJ, Johannes KJ. Randomised placebo-controlled double blind trial on "essential" phospholipids in the treatment of fatty liver associated with diabetes. Méd Chir Dig. 1988;17(1):61-65.

14. Yin D, Kong L. Observation for curative effect of Essentiale in treatment of fatty liver caused by diabetes mellitus. Med $J$ Q Ilu. 2000;15:277-278.

15. Poongothai S, Karkuzhali K, Siva Prakash G, et al. Effect of essentiale in diabetic subjects with non-alcoholic fatty liver. Int J Diab Dev Ctries. 2005;25(1):12-19.

16. Ohbayashi H, Fujimoto M, Yamase H, Ito M. Improvement of NASH with two-year treatment with oral polyenephosphatidylcholine. J Rural Med. 2007;1:67-73.

17. Buyeverov AO, Yeshanu VS, Mayevskaya MV, Ivashkin VT. Application of essential phospholipids in complex therapy of steatohepatitis of the mixed origin. Klin Persp Gastroenterol Hepatol. 2008;1:17-22. Russian.

18. Sun C, Zheng X, Tan Z, Cui F, Zhang R, Zhang H. Clinical observation on polyene phosphatidyl choline and metformin in the treatment of type 2 diabetes and non-alcoholic fatty liver disease. Clin Focus. 2008;23(17):1272-1273. Chinese.

19. Sas E, Grinevich V, Efimov O, Shcherbina N. Beneficial influence of polyunsaturated phosphatidylcholine enhances functional liver condition and liver structure in patients with nonalcoholic steatohepatitis accompanied by diabetes type 2 . Results of prolonged randomized blinded prospective clinical study [abstract]. J Hepatol. 2013;58:S549.

20. Ohbayashi H. Twelve-month chronic administration of polyenephosphatidylcholine $\left(\mathrm{EPL}^{\circledR}\right)$ for improving hepatic function of fatty liver patients. Prog Med. 2004;24(7):1751-1756. Japanese.

21. Ohbayashi H, Fujimoto M, Yoshida M, Ito M, Yamase H. The therapeutic effect of polyenephosphatidylcholine (EPL) on NASH. Liver Bile Pancreas. 2006;52(4):637-642. Japanese.

22. Liang H. Treatment of fatty liver applying polyene phosphatidyl choline. Chin Med Factory Mine. 2006;19(3):207-208. Chinese.

23. Fan X, Deng Y, Ye L, et al. Effect of Xuezhikang capsule on serum tumor necrosis factor- $\alpha$ and interleukin- 6 in patients with nonalcoholic fatty liver disease and hyperlipidemia. Chin J Integr Med. 2010;16(2):119-123.

24. Guo XL, Liang PX, Xu YQ. Comparison of efficacies of diammonium glycyrrhizinate and polyene phosphatidylcholine on nonalcoholic steatohepatitis. Chin J New Drugs. 2012;21(3):289-293. Chinese.

25. Shen Z. Observation of efficacy in 100 patients with non-alcoholic fatty liver disease treated with Essentiale. Chin J Curr Clin Med. 2007;5(8):693-694. Chinese.

26. Arvind N, Savaikar P, Rajkumar JS. Therapy for NAFLD - comparative study of essential phospholipids vs ursodeoxycholic acid. Ind J Clin Pract. 2006;16(10):21-24.

27. Zhuang XS, Zhang ZJ. Study of ursodeoxycholic acid (UDCA) combined with polyene phosphatidylcholine in the treatment of non-alcoholic steatohepatitis. China Pract Med. 2009;4(10):11-12. Chinese. 
28. Zhou SY, Sun ZY. Therapeutic efficacy of polyunsaturated phosphatidylcholine on fatty liver disease. J Clin Hepatol. 2010;26(3):286-287. Chinese.

29. Hu G, Liu K, Zhao L. Polyunsaturated phosphatidylcholine (Essentiale) for alcoholic/fatty liver: a systematic review. Liver. 2005;10(1):5-7. Chinese.

30. Panos MZ, Polson R, Johnson R, Portmann B, Williams R. Polyunsaturated phosphatidyl choline for acute alcoholic hepatitis: a double-blind, randomized, placebo-controlled trial. Eur J Gastroenterol Hepatol. 1990;2(5):351-355.

31. Lieber CS, Weiss DG, Grozmann R, Paronetto F, Schenker S, Veterans Affairs Cooperative Study 391 Group. II. Veterans affairs cooperative study of polyenylphosphatidylcholine in alcoholic liver disease. Alcohol Clin Exp Res. 2003;27(11):1765-1772.

32. Knüchel F. Double-blind study in patients with alcoholic fatty liver. The influence of "essential" phospholipids on enzyme behaviour and lipid composition of the serum [Doppelblindstudie bei Patienten mit alkoholtoxischer Fettleber. Der Einfluß von “essentiellen” Phospholipiden auf Enzymverhalten und Lipidzusammensetzung des Serums bei Patienten mit alkoholtoxischer Fettleber]. Med Welt. 1979;30(11):411-416. German.

33. Schüller Pérez A, Gonzáles San Martin F. Placebo-controlled study with polyunsaturated phosphatidylcholine in alcoholic steatosis of the liver. Med Welt. 1985;36(16):517-521.

34. Lieber CS. New concepts of the pathogenesis of alcoholic liver disease lead to novel treatments. Curr Gastroenterol Rep. 2004;6(1):60-65.

35. Sas E, Grinevich V, Kravchuk U, Efimov O. Polyunsaturated phosphatidylcholine reduces insulin resistance and hepatic fibrosis in patients with alcoholic liver disease: results of randomized blinded prospective clinical study [abstract]. J Hepatol. 2011;54:S207.
36. Gundermann KJ, Lehmacher W. The essential phospholipids as liver therapeutic - a meta-analysis of double-blind trials in chronic liver disease [Niezbędne fosfolipidy w leczeniu choró wątroby - metaanaliza podwójnie ślepych prób klinicznych w przewlekłych chorobach wątroby]. Gastroenterol Polska. 1998;5(6):553-559. Polish.

37. Adams LA, Angulo P. Treatment of non-alcoholic fatty liver disease. Postgrad Med J. 2006;82(967):315-322.

38. Ling J, Chaba T, Zhu LF, Jacobs RL, Vance DE. Hepatic ratio of phosphatidylcholine to phosphatidylethanolamine predicts survival after partial hepatectomy in mice. Hepatology. 2012;55(4):1094-1102.

39. International Diabetes Federation [homepage on the Internet]. The IDF Consensus Worldwide Definition of the Metabolic Syndrome; 2006. Available from: http://www.idf.org/. Accessed March 15, 2016.

40. Klinger W, Gundermann KJ. (3-sn-phosphatidyl)choline in hyperlipoproteinemia [3-sn-Phosphatidyl)cholin bei Hyperlipoproteinämien]. Dtsch Ärztebl. 1992;26(Suppl):24-25. German.

41. Kirsten R, Heintz B, Nelson K, et al. Polyenylphosphatidylcholine improves the lipoprotein profile in diabetic patients. Int $\mathrm{J}$ Clin Pharmacol Ther. 1994;32(2):53-56.

42. Okiyama W, Tanaka N, Nakajima T, et al. Polyenephosphatidylcholine prevents alcoholic liver disease in PPAR $\alpha$-null mice through attenuation of increases in oxidative stress. J Hepatol. 2009;50(6):1236-1246.

43. Lieber CS, Weiss DG, Groszmann R, Paronetto F, Schenker S, Veterans Affairs Cooperative Study 391 Group. I. Veterans Affairs Cooperative Study of polyenylphosphatidylcholine in alcoholic liver disease: effects on drinking behavior by nurse/physician teams. Alcohol Clin Exp Res. 2003;27(11):1757-1764.
Clinical and Experimental Gastroenterology

\section{Publish your work in this journal}

Clinical and Experimental Gastroenterology is an international, peerreviewed, open access journal, publishing all aspects of gastroenterology in the clinic and laboratory, including: Pathology, pathophysiology of gastrointestinal disease; Investigation and treatment of gastointestinal disease; Pharmacology of drugs used in the alimentary tract;

\section{Dovepress}

Immunology/genetics/genomics related to gastrointestinal disease. This journal is indexed on CAS. The manuscript management system is completely online and includes a very quick and fair peer-review system. Visit http://www.dovepress.com/testimonials.php to read real quotes from published authors.

Submit your manuscript here: http://www.dovepress.com/clinical-and-experimental-gastroenterology-journal 